\title{
Free Energy Landscape and Folding Mechanism of a $\beta$ - Hairpin in Explicit Water: A Replica Exchange Molecular Dynamics Study
}

\author{
Phuong H. Nguyen, ${ }^{1 *}$ Gerhard Stock, ${ }^{1}$ Emil Mittag, ${ }^{2}$ Chin-Kun Hu, ${ }^{3}$ and Mai Suan Li ${ }^{4 *}$ \\ ${ }^{1}$ Institute of Physical and Theoretical Chemistry, J.W. Goethe University, Frankfurt, Germany \\ ${ }^{2}$ Center for Bioinformatics Hamburg, University of Hamburg, Hamburg, Germany \\ ${ }^{3}$ Institute of Physics, Academia Sinica, Taipei, Taiwan, and National Center for Theoretical Sciences at Taipei, Physics \\ Division, National Taiwan University, Taipei, Taiwan \\ ${ }^{4}$ Institute of Physics, Polish Academy of Sciences, Warsaw, Poland
}

ABSTRACT The free energy landscape and the folding mechanism of the C-terminal $\beta$-hairpin of protein $G$ is studied by extensive replica exchange molecular dynamics simulations (40 replicas and 340 ns total simulation time), using the GROMOS96 force field and the SPC explicit water solvent. The study reveals that the system preferentially adopts a $\beta$-hairpin structure at biologically important temperatures, and that the helix content is low at all temperatures studied. Representing the free energy landscape as a function of several types of reaction coordinates, four local minima corresponding to the folded, partially folded, molten globule, and unfolded states are identified. The findings suggest that the folding of the $\beta$-hairpin occurs as the sequence: collapse of hydrophobic core $\rightarrow$ formation of $\mathrm{H}$-bond $\rightarrow$ formation of the turn. Identifying the folded and molten globule states as the main conformations, the free energy landscape of the $\beta$-hairpin is consistent with a two-state behavior with a broad transition state. The temperature dependence of the folding-unfolding transition is investigated in some detail. The enthalpy and entropy jumps at the folding transition temperature are found to be about three times lower than the experimental estimates, indicating that the folding-unfolding transition in silico is less cooperative than its in vitro counterpart. Proteins 2005;61:795-808.

$\odot 2005$ Wiley-Liss, Inc.

Key words: $\beta$-hairpin; folding mechanism; principal component analysis; replica exchange simulation

\section{INTRODUCTION}

Understanding the dynamics and mechanism of protein folding remains one of the most challenging problems in molecular biology. ${ }^{1}$ As a suitable model system to study the folding of $\beta$-sheets in isolation, the 16 -residue $\beta$-hairpin from the $\mathrm{C}$-terminus of protein $\mathrm{G}$ has been investigated in the pioneering experiments by Serrano ${ }^{2,3}$ and Eaton. ${ }^{4}$ These works have inspired several molecular dynamics (MD) simulations on this system using both implicit and explicit solvent models, ${ }^{5-18}$ because understanding of the folding of key protein structures may provide a basis for the understanding of more complicated globular proteins. Despite this effort, the mechanism, pathways, and cooperativity of the folding of $\beta$-hairpin remain a controversial issue.

To our knowledge, there exist three possible scenarios of folding for the $\beta$-hairpin. The first one is a "hydrogen bond zipping" mechanism, in which folding is initiated at the turn and propagates towards the tails. The hydrophobic clusters, which play a crucial role in stabilizing the system, form relatively late in this scenario. Employing a helix coil-type model, this mechanism was predicted by Eaton et al., ${ }^{19}$ and is also favored by recent experiments of $\mathrm{Du}$ et al. ${ }^{20}$ The zipping mechanism is supported by a off-lattice simulation of Klimov and Thirumalai, ${ }^{21}$ but is in variance to all-atom simulations using the CHARMM, ${ }^{5}$ AMBER,${ }^{10}$ and OPLS-AA ${ }^{11}$ force fields, respectively. Instead, in the latter a second scenario was proposed, the so-called "hydrophobic-core-centric" mechanism. Following an initial core collapse and before folding into the native state, in this scenario the peptide rapidly adopts a partially folded state $\mathrm{P}$ with two to three hydrogen bonds. Inspired by the experiments of Honda et al. ${ }^{22}$ and Kobayashi et al., ${ }^{23}$ and by using the AGBNP implicit solvent model, Felts et al..$^{15}$ have obtained evidence for a third folding mechanism, which is a hybrid of the first and second one. That is, folding is initiated neither by zipping nor by hydrophobic collapse, but it rather is a cooperative

Grant sponsor: the Polish KBN; Grant number: 1P03B01827; Grant sponsor: National Science Council in Taiwan; Grant number: NSC 93-2112-M 001-027, 94-2119-M-002-001; Grant sponsor: Academia Sinica in Taiwan; Grant number: AS-92-TP-A09; Grant sponsor: the Frankfurt Center for Scientific Computing; Grant sponsor: the Deutsche Forschungsgemeinschaft.

*Correspondence to: Mai Suan Li, Institute of Physics, Polish Academy of Science, A1. Lotnikow 32/46, 02-668 Warsaw, Poland. E-mail: masli@ifpan.edu.pl or Phuong H. Nguyen, Institute of Physical and Theoretical Chemistry, J. W. Goethe University, Marie-CurieStr. 11, D-60439 Frankfurt, Germany. E-mail: phuong@theochem.unifrankfurt.de

Received 4 March 2005; Revised 22 June 2005; Accepted 28 June 2005

Published online 20 October 2005 in Wiley InterScience (www.interscience.wiley.com). DOI: 10.1002/prot.20696 
process that involves the nearly simultaneous formation of the hydrophobic core, hydrogen bonds, and the turn.

Another issue of debate concerns the possible existence of helical folding intermediates of the $\beta$-hairpin. Using the AMBER94 force field, Garcia and Sanbonmatsu ${ }^{10}$ have found that the $\beta$-hairpin spends a considerable amount of time in the helix-rich phase, even at low temperatures. Zagrovic et al. ${ }^{9}$ have also found significant helical intermediates at $300 \mathrm{~K}$ with the OPLS united-atom force field. However, experimental ${ }^{2,4}$ and several theoretical studies $^{5,6,12,16-18}$ have not found significant helical content. Furthermore, the thermodynamics of the $\beta$-hairpin folding has been considered. From tryptophan fluorescence experiments Munoz et al. ${ }^{4}$ estimated the thermodynamic parameters for the folding transition to be $\Delta H=48.5 \mathrm{~kJ} / \mathrm{mol}$, $\Delta S=163.2 \mathrm{~J} /(\mathrm{mol} \mathrm{K})$, and a folding transition temperature $T_{F}=297 \mathrm{~K}$. Previous all-atom simulations underestimate $\Delta H$ and $\Delta S$, and therefore overestimate $T_{F}$. Depending on the model and the simulation conditions, the value of $T_{F}$, for example, ranges from $330 \mathrm{~K}^{10}$ to $485 \mathrm{~K}^{15}$ Very recently, Yoda et al. ${ }^{17,18}$ presented multicanonical replicaexchange MD simulations using various force fields. Their results suggest that the above discrepancies are at least partly caused by the different force fields under consideration.

In this work, the free energy landscape and the folding mechanism of the $\mathrm{C}$-terminal $\beta$-hairpin of protein $\mathrm{G}$ is studied by replica exchange $\mathrm{MD}$ simulations, ${ }^{24-31}$ using the GROMOS96 force field $43 \mathrm{a} 1^{32}$ and the SPC water model. ${ }^{33}$ The study reveals a preference for the second mechanism of folding, and shows that the helix content is low at all temperatures studied. Representing the free energy landscape of the peptide as a function of several types of reaction coordinates, we find that the free energy landscape of the $\beta$-hairpin is consistent with a two-state behavior with a broad transition state. The advantage of our study compared to the previous work is that we study the folding mechanism in much more detail using the free energy landscape approach. Present simulations give $T_{F}=$ $389 \mathrm{~K}$ and values of $\Delta H$ and $\Delta S$ about three times smaller than the experimental ones. Also, we find that the cooperativity index for the folding-unfolding transition is about an order of magnitude lower than the experimental estimate. So similar to other force fields the GROMOS force field does not provide reasonable estimates for thermodynamic parameters. However, it gives a reasonable estimation not only for the folding time but also for the population of $\beta$-hairpin at the biologically relevant temperature interval. Finally, the problems of current theoretical models to reproduce the high-temperature range are discussed in some detail.

\section{Model and MD Simulation}

\section{MODELS AND METHODS}

We used the GROMOS96 force field $43 \mathrm{a}^{32}$ to model the 16 -residue $\beta$-hairpin peptide and the SPC water model ${ }^{33}$ to describe the solvent. Starting with the C-terminal fragment (residues 41-56) of the protein G (PDB ID code 2gb1), the 16-residue $\beta$-hairpin is capped with the Ace and
Nme groups, resulting in a blocked peptide sequence of Ace-GEWTYDDATKTFTVTE-Nme. The peptide was placed in a cubic box containing 1321 water molecules.

In all simulations, the GROMACS program suite ${ }^{34,35}$ was employed. The equations of motion were integrated by using a leap-frog algorithm with a time step of 2 fs. Covalent bond lengths were constrained via the $\mathrm{SHAKE}^{36}$ procedure with a relative geometric tolerance of $10^{-4}$. We used the particle-mesh Ewald method to treat the longrange electrostatic interactions. ${ }^{37}$ The nonbonded interaction pair-list were updated every $5 \mathrm{fs}$, using a cutoff of 1.2 $\mathrm{nm}$.

The peptide was minimized using the steepest decent method. Subsequently, the solvated system was equilibrated for $100 \mathrm{ps}$ at constant pressure (1 atm) and temperature $(T=300 \mathrm{~K})$, respectively, using the Berendsen coupling procedure. ${ }^{38}$ The system was then equilibrated further at constant temperature $(T=300 \mathrm{~K})$ and constant volume.

\section{Replica-Exchange Method}

The replica exchange or parallel tempering method m $^{24-31}$ has been proposed to study systems with a rugged energy landscape. Its Monte Carlo and molecular dynamics versions were applied by Hansmann ${ }^{28}$ to biomolecules for the first time. Here we employ the version of the replica exchange molecular dynamics (REMD), which was described in detail elsewhere. ${ }^{29,31}$ In brief, the method constructs a generalized ensemble from $M$ noninteracting trajectories with temperatures $T_{\mathrm{m}}(m=1, \ldots, M)$. A state of this generalized ensemble is characterized by $X=$ $\left\{\ldots, x^{(\mathrm{i})}{ }_{m} \ldots\right\}$, where $x^{(\mathrm{i})}{ }_{m}$ represents the coordinates $q^{(\mathrm{i})}{ }_{m}$ and momenta $p^{(\mathrm{i})}{ }_{m}$ of all atoms of the ith replica at temperature $T_{m}$. The algorithm consists of two consecutive steps: (1) independent constant-temperature simulations of each replica, and (2) exchange of the trajectories (i.e., the coordinates) between the replicas (defined by the temperature) according to a Metropolis-like criterion. Considering, for example, the exchange $X=\left\{\ldots, x^{(\mathrm{i})}{ }_{m}, \ldots, x^{(\mathrm{j})}{ }_{n}, \ldots\right\}$ $\rightarrow X^{\prime}=\left\{\ldots, x^{(\mathrm{j})}{ }_{m}, \ldots, x^{(\mathrm{i})}{ }_{n}, \ldots\right\}$, the criterion is given by

$$
\begin{gathered}
W\left(x_{m}^{(i)}, x_{n}^{(j)}\right)=\left\{\begin{array}{c}
\exp (-\Delta) \text { if } \Delta>0(1) \\
1 \text { if } \Delta \leq 0
\end{array}\right. \\
\Delta=\left[E\left(q^{(j)}\right)-E\left(q^{(i)}\right)\right]\left[\left(k_{B} T_{m}\right)^{-1}-\left(k_{B} T_{n}\right)^{-1}\right],
\end{gathered}
$$

where $E\left(q^{(i)}\right)$ and $E\left(q^{(j)}\right)$ are the potential energies of the $i$ th and $j$ th replica, respectively. After the exchange, the momenta $\underline{p}^{\prime(i)}$ and $p^{\prime(j)}$ of the replicas are given by

$$
p^{\prime(i)}=\sqrt{\frac{T_{n}}{T_{m}}} p^{(i)}, p^{\prime(j)}=\sqrt{\frac{T_{m}}{T_{n}}} p^{(j)} .
$$

Iterating steps (1) and (2), the trajectories of the generalized ensemble perform a random walk in temperature space. This facilitates an efficient conformational sampling of the energy landscape of the system, even in the presence of multiple local minima. To obtain the canonical average of any physical quantity at a specific temperature, one performs a time average over all trajectory segments of the replica corresponding to this temperature. To inter- 
face the REMD algorithm with the GROMACS program package, an external shell script was employed that controls the $M$ independent GROMACS trajectories of the REMD simulation.

To achieve the optimal performance of the algorithm for a given system, the temperatures of the replicas are chosen such (1) that the lowest temperature is small enough to sufficiently sample the states of low energy, (2) that the highest temperature is large enough to overcome energy barriers of the system, and (3) that the acceptance ratio of criterion (1) is sufficiently high. In the case of the $\beta$-hairpin in water described below, we therefore employed the procedure suggested in refs. 39 and 40 , and run several short simulations at temperatures $T=280,320,370,400$, 460 , and $520 \mathrm{~K}$. From these simulations, we obtained the total potential energy $E$ as a function of temperature $T$. Requesting an acceptance ratio of $20-30 \%$, the temperature distribution for the replicas was then obtained by solving Equation (2) iteratively. This way, we obtained a total of 40 temperatures from $270 \mathrm{~K}$ to $538 \mathrm{~K}$, with a gap of 4-6 $\mathrm{K}$ between adjacent replicas.

Employing the structure equilibrated at $300 \mathrm{~K}$ as a starting structure for each of the 40 replicas, each replica was run independently at its own temperature for 200 ps. The exchange procedure between the replicas was then activated, using a time step of 1.5 ps between any two exchange attempts. This time interval should be large enough compared to the coupling time of the heat bath $(\tau=$ $0.1 \mathrm{ps})$ such that the trajectory is roughly equilibrated before the next exchange is attempted. Each replica was run for $8.6 \mathrm{~ns}$. The data were collected every $0.1 \mathrm{ps}$. To obtain the canonical average of any physical quantity at a specific temperature, one performs a time average over all trajectory segments of the replica corresponding to this temperature.

\section{Principal Component Analysis}

Principal component analysis (PCA) is an efficient method to represent the conformational distribution of a 3 $N$-dimensional system in terms of a few "principal" components.41-44 The basic idea is that the correlated internal motions are represented by the covariance matrix

$$
\sigma_{i j}=\left\langle\left(q_{i}-\left\langle q_{i}\right\rangle\right)\left(q_{j}-\left\langle q_{j}\right)\right\rangle\right\rangle,
$$

where $q_{1}, \ldots q_{3 N}$ are the mass-weighted Cartesian coordinates of the solute molecule and $\langle\ldots\rangle$ denotes the average over all sampled conformations. By diagonalizing $\sigma$, we obtain $3 N$ eigenvectors $v_{n}$ and eigenvalues $\lambda_{n}$, which are rank-ordered in descending order, that is, $\lambda_{1}$ represents the largest eigenvalue. The eigenvectors and eigenvalues of $\sigma$ yield the modes of collective motion and their amplitudes. It has been shown that a large part of the system's fluctuations can be described in terms of only a few principal components. ${ }^{41-44}$

In addition to the standard PCA (cPCA) described above, we also performed a PCA based on the $(\phi, \psi)$ dihedral angles of the peptide. ${ }^{45}$ For flexible systems such as folding peptides, the use of internal coordinates may be advantageous, because problems associated with the mixing of internal and overall motions are circumvented. To uniquely define the distance in the space of periodic dihedral angles, the dihedral PCA (dPCA) employs the variables ${ }^{45}$

$$
q_{k}=\cos \left(\alpha_{k}\right), q_{k+1}=\sin \left(\alpha_{k}\right),
$$

where $\alpha_{k} \in\left\{\phi_{k}, \psi_{k}\right\}$ and $k=1 \ldots N-1$, with $N$ being the number of backbone and side-chain dihedral angles.

\section{Free Energy Landscape}

To explore the thermodynamics and kinetics of folding, we calculated the free energy as a function of several types of reaction coordinates $v_{i}$, including (1) the number of native hydrogen bonds (H-bonds) in the $\beta$-hairpin conformation, (2) the radius of gyration of the hydrophobic core $R_{g}{ }^{\text {core }}$, (3) the enthalpic energy $E$, (4) the root-mean-square distance (RMSD) from the PDB structure, (5) and various principal components. The corresponding free energy surface along the $n$-dimensional reaction coordinate $V=$ $\left(v_{1}, \ldots v_{n}\right)$ is given by

$$
\Delta G(V)=-k_{B} T\left[\ln P(V)-\ln P_{\max }\right],
$$

where $P(V)$ is the probability distribution obtained from a histogram of the MD data.

$P_{\max }$ denotes the maximum of the distribution, which is subtracted to ensure that $\Delta G=0$ for the lowest free energy minimum.

\section{Structural Susceptibility}

To study the probability of being in the native state, we use the following overlap function ${ }^{46}$

$$
\chi=\frac{1}{n_{\text {total }}} \sum_{i<j+1}^{N} \theta\left(d_{c}-r_{i j}\right) \Delta_{i j}
$$

where $n_{\text {total }}$ is the total number of native contacts, $r_{i j}$ is the distance between atoms $i$ and $j$, and $\Delta_{i j}$ is equal to 1 if atoms $i$ and $j$ form a native contact, and 0 otherwise. A native contact is said to be formed if $r_{i j}{ }^{0} \leq d_{c}$, where $r_{i j}{ }^{0}$ is the distance between atoms $i$ and $j$ in the native conformation. Being one if the conformation of the polypeptide chain coincides with the native structure and zero for unfolded conformations, the overlap function $\chi$ serves as an order parameter for the folding-unfolding transition. The probability of being in the native state, $f_{N}$, which can be measured by various experimental techniques, is defined as $f_{N}=\langle\chi\rangle$, where $\langle\ldots\rangle$ stands for a thermal average. To calculate $\chi$, we only considered the positions of the $\alpha$-carbons. We performed this calculation with different values of the cutoff distance $\left(d_{c}=5.5,6.0,6.5,7.0,7.5,8.0\right.$ and 8.5 $\AA$ ), to check if the thermodynamic quantities depend on its choice. The folding transition temperature $T_{F}$ is defined as the maximum of the structural susceptibility ${ }^{46}$

$$
\Delta \chi=\left\langle\chi^{2}\right\rangle-\langle\chi\rangle^{2} .
$$

\section{Equilibration Procedure}

\section{RESULTS}

To demonstrate the performance of the REMD algorithm, Figure 1 shows the time evolution of exchange 

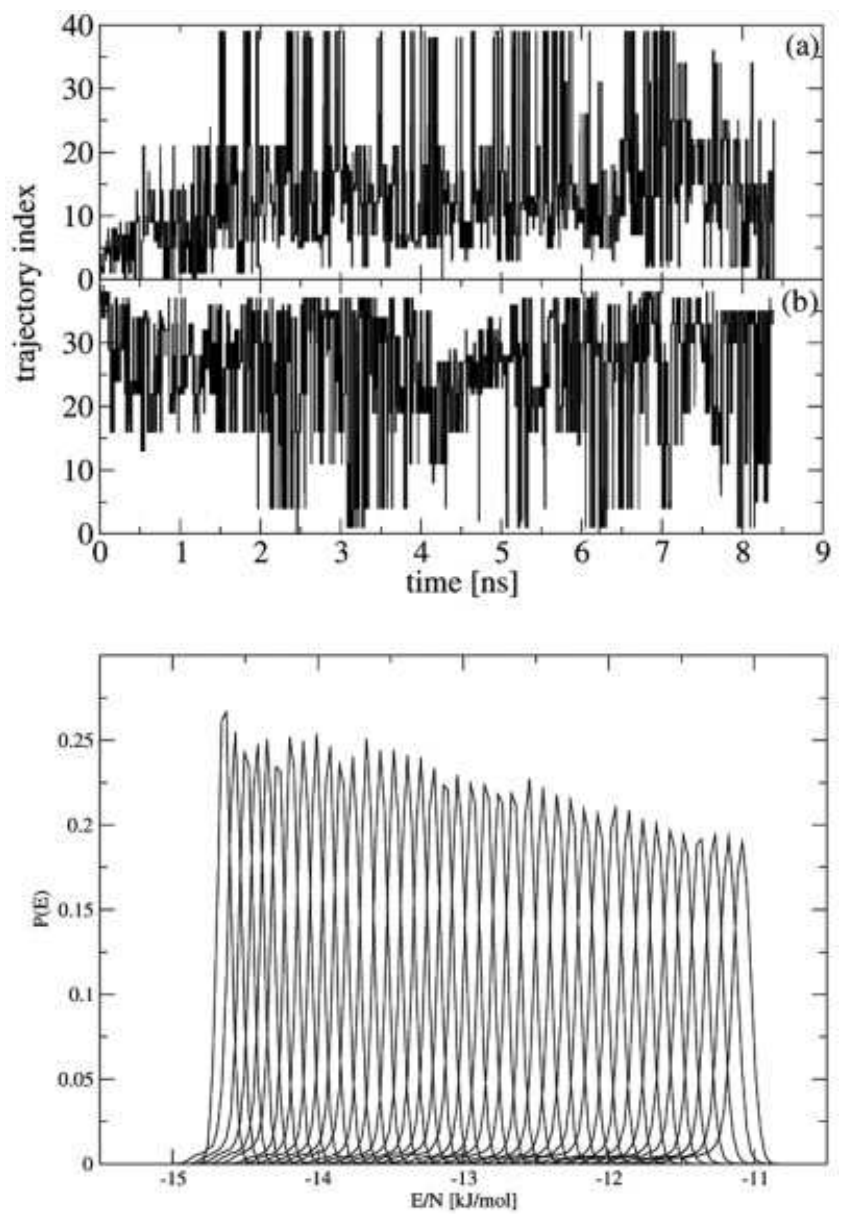

Fig. 1. Performance of the replica exchange method for the $\beta$-hairpin. The upper panel demonstrates the random walk in temperature space of the simulation, as monitored by the index of the replica, which is exchanged with the (a) first and (b) 40th replica, respectively. The lower panel shows the distribution of the ptoential energy per atom for all the replicas.

partners of a specific replica, adopting the first and the 40th replicas of the simulation as representative examples. Clearly, during the course of the simulation each replica is visited many times by each trajectory, or in other words, each trajectory performs a random walk in temperature space. Furthermore, Figure 1 shows the distribution of the potential energy per atom for all of the replicas. As the temperature increases they become more and more broad. For adjacent temperatures the corresponding distributions have enough overlap, which guarantees reasonable exchange probabilities (20-30\%).

To check the convergence of the REMD simulations with respect to the sampling of conformational space, we compared the structural susceptibility shown in Figure 8 to results obtained from 5-ns (instead of 8.6-ns) simulations (data not shown). Only minor changes were observed in this comparison. Further evidence for sufficient equilibration is provided by Yoda et al., ${ }^{17,18}$ who also studied the secondary structure formation by the GROMOS96 force field. However, their work is different from ours in two aspects. First, they used the multicanonical replica- exchange method, and second, more importantly, their simulations start from random configurations but not from the native ones as it has been done in the present work and in other articles. The advantage of using initial random structures is that it allows one to observe direct formation of secondary structures and avoid possible overestimation of their content due to starting native structures. Despite these differences, our results for the $\alpha$-helical and $\beta$-sheet contents shown in Figure 4 are consistent with those of Yoda et al. ${ }^{17,18}$ This agreement suggests that both our data and data of these authors are sufficiently equilibrated.

\section{Mechanism of Folding}

A wealth of information on the folding mechanism can be extracted from the free energy landscape of the $\beta$-hairpin. Several authors have shown that the number of hydrogen bonds (H-bonds) and the radius of gyration of the hydrophobic core $R_{g}{ }^{\text {core }}$ play an important role in elucidating the folding process of the $\beta$-hairpin peptide. ${ }^{5,10,11,16}$ Hence, Figure 2 shows the free energy landscape as a function of these two reaction coordinates, where the hydrophobic core is formed by the side chains of the residues Trp3, Tyr5, Phe12, and Val14. At $T=300 \mathrm{~K}$, there are four local minima corresponding to the folded $(\mathrm{F})$, partially folded $(\mathrm{P})$, molten globule $(\mathrm{H})$, and unfolded (U) states. With increasing temperature, the global minimum $\mathrm{F}$ becomes more and more shallow and almost disappears at $362 \mathrm{~K}$.

Before discussing the folding mechanism, we first characterize the structure of the conformational states $\mathrm{U}, \mathrm{H}, \mathrm{P}$, and $\mathrm{F}$ at $300 \mathrm{~K}$. Table I shows the population, the mean number of $\mathrm{H}$-bonds, the mean hydrophobic-core radius of gyration $\left\langle R_{g}{ }^{\text {core }}\right\rangle$, as well as the mean dihedral angles $(\phi, \psi)$ of the 16 peptide groups. On average, the folded state $\mathrm{F}$ has 4 to 6 (out of 7) H-bonds. State P has a compact hydrophobic core with $R_{g}{ }^{\text {core }} \approx 5 \AA$ and 2 to $3 \beta$-strand H-bonds. The molten globule state $\mathrm{H}$ remains compact, but has only zero to one H-bonds. In terms of the mean backbone dihedral angles, the structures of three residues at the turn Asp7, Ala8, and Thr9 appear to be quite different for the U and other states, while the remaining residues have a similar structure, that is the $\beta$-sheet structure. Interestingly, the residue Ala8 of the unfolded state $U$ is found in $\alpha_{R}$-helical conformation, whereas it is in $\alpha_{L}$-helical conformation for other states. The state $\mathrm{P}$ differs from the state $\mathrm{H}$ mainly at residue Thr9, while it differs from state $\mathrm{F}$ primarily at residues Thr9 and Lys10. The collapse of the hydrophobic core mainly affects the structure of residues Tyr5 and Phe12, that is, the $\psi$ angles of these residues undergo a large change upon going from the state $\mathrm{U}$ to the others.

Because the free energy contours shown in Figure 2 have an L-shape and because there is only a small barrier between the $\mathrm{U}$ and the $\mathrm{H}$ state, the folding proceeds according to Zhou et al. ${ }^{11,16}$ via the hydrophobic corecentric mechanism. A more "diagonal" shape would correspond to the zipping or hydrophobic bond-centric scenario. However, we observe a notable difference in population of states $\mathrm{H}$ and $\mathrm{P}$, compared to Zhou et al., ${ }^{11}$ who showed that 

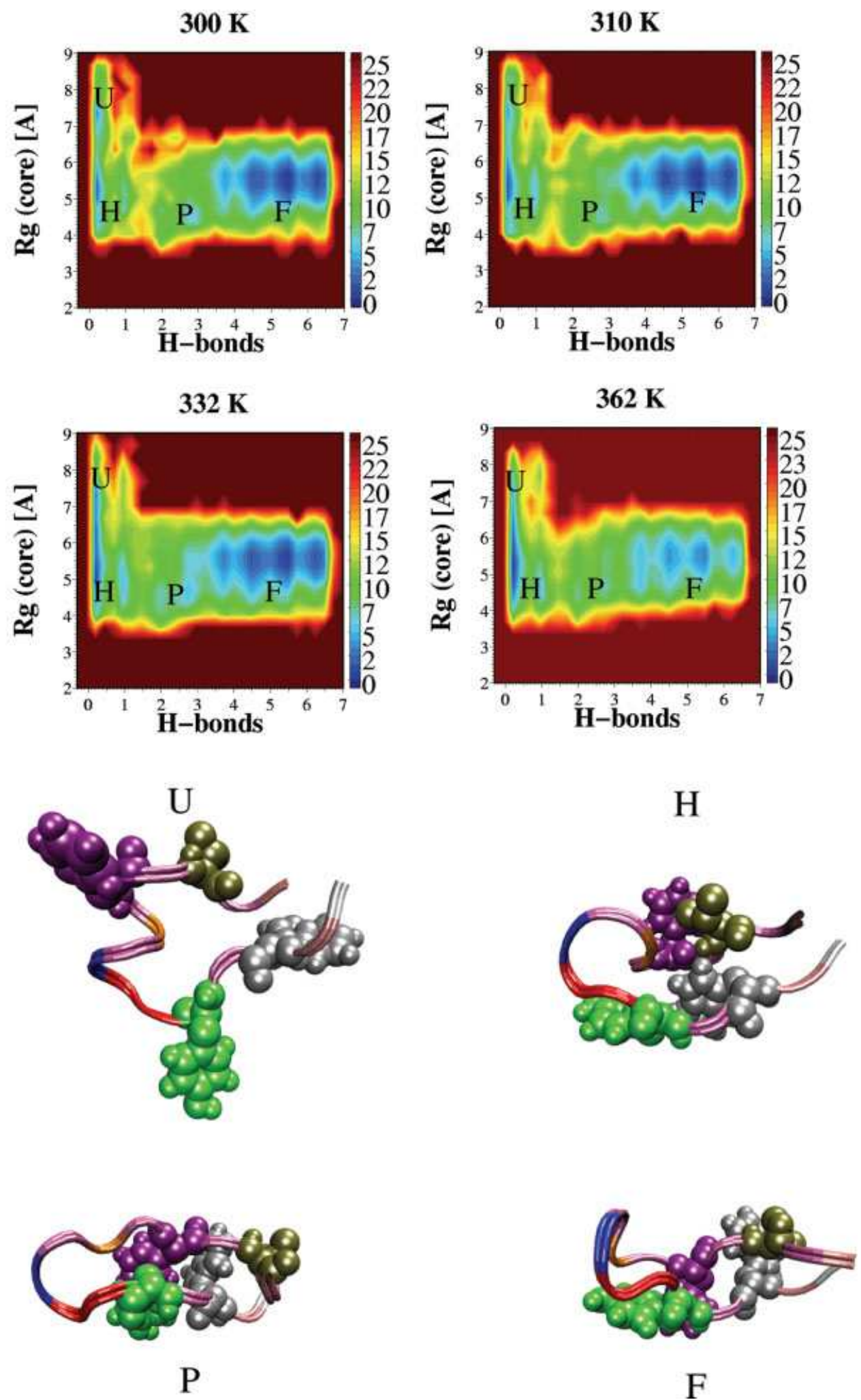

$\mathrm{F}$

Fig. 2. Free energy map (in $\mathrm{kJ} / \mathrm{mol}$ ) at $300,310,332$, and $362 \mathrm{~K}$, plotted as a function of the number of $\mathrm{H}$ bonds and the hydrophobic-core radius of gyration $R_{g}^{c o r e}$. Typical structures for the $U, H, P$, and $F$ conformational states are shown for $T=300 \mathrm{~K}$. 
TABLE I. Comparison of the Conformational States of the $\beta$-Hairpin as Revealed by the Free-Energy Surfaces $\Delta G\left(\right.$ H-bond, $R_{g}{ }^{\text {core })}$ and $\Delta G($ RMSD, $E)$ displayed in Figures 2 and 5, Respectively

\begin{tabular}{|c|c|c|c|c|c|c|c|}
\hline \multirow{2}{*}{$\begin{array}{l}\text { Map } \\
\text { State }\end{array}$} & \multicolumn{4}{|c|}{$\mathrm{HB}-R_{g}$} & \multicolumn{3}{|c|}{ RMSD- $E$} \\
\hline & $\mathrm{U}$ & $\mathrm{H}$ & $\mathrm{P}$ & $\mathrm{F}$ & $\mathrm{F}^{\prime}$ & $\mathrm{H}^{\prime}$ & $\mathrm{U}^{\prime}$ \\
\hline Population (\%) & 3.5 & 11.3 & 3.9 & 55.3 & 69.8 & 25.4 & 4.7 \\
\hline$\langle\mathrm{HB}\rangle$ & 0.09 & 0.2 & 2.6 & 5.1 & 4.7 & 0.4 & 0.3 \\
\hline$\left\langle R_{g}^{\text {core }}\right\rangle$ & 7.7 & 5.2 & 5.1 & 5.0 & 5.5 & 6.2 & 6.1 \\
\hline Gly1 & $-46,54$ & $-6,25$ & $3,-25$ & $22,-31$ & $46,-42$ & $-4,26$ & $31,-96$ \\
\hline Glu2 & $-52,106$ & $-86,102$ & $-93,122$ & $-102,122$ & $-103,133$ & $-84,100$ & $-79,20$ \\
\hline Trp3 & $-85,112$ & $-109,130$ & $-89,118$ & $-87,106$ & $-82,107$ & $-104,139$ & $-121,142$ \\
\hline Thr4 & $-94,135$ & $-75,102$ & $-99,123$ & $-115,136$ & $-119,138$ & $-78,113$ & 3,68 \\
\hline Tyr5 & $-77,36$ & $-83,100$ & $-109,116$ & $-94,133$ & $-91,131$ & $-83,54$ & $-110,7$ \\
\hline Asp6 & $-94,31$ & $-61,121$ & $-94,132$ & $-107,114$ & $-103,118$ & $-63,128$ & $-39,109$ \\
\hline Asp7 & $-91,32$ & $-62,-3$ & $-63,-35$ & $-61,-42$ & $-60,-41$ & $-74,14$ & $-4,-45$ \\
\hline Ala8 & $2,-41$ & $-71,-35$ & $-80,-46$ & $-64,-46$ & $-67,-46$ & $-56,-43$ & $-75,25$ \\
\hline Thr9 & $-81,56$ & $-91,-4$ & $-97,51$ & $-83,-12$ & $-86,4$ & $-85,-25$ & $-72,23$ \\
\hline Lys10 & $-84,31$ & $-64,66$ & $-26,60$ & 52,45 & 33,45 & $-65,56$ & $-89,27$ \\
\hline Thr11 & $-88,53$ & $-94,48$ & $-109,104$ & $-121,131$ & $-125,127$ & $-86,27$ & $-98,97$ \\
\hline Phe12 & $-70,42$ & $-85,99$ & $-68,101$ & $-100,112$ & $-90,108$ & $-92,76$ & $-54,-44$ \\
\hline Thr13 & $-98,33$ & $-82,50$ & $-108,110$ & $-117,134$ & $-118,127$ & $-94,99$ & $-80,-56$ \\
\hline Val14 & $-91,132$ & $-109,104$ & $-75,106$ & $-84,111$ & $-76,105$ & $-100,131$ & $-104,-47$ \\
\hline Thr15 & $-59,56$ & $-79,46$ & $-57,64$ & $-99,100$ & $-103,101$ & $-66,51$ & $-95,98$ \\
\hline Glu16 & $-100,-58$ & $-96,-58$ & $-90,-59$ & $-81,-58$ & $-71,-58$ & $-97,-58$ & $-100,-59$ \\
\hline
\end{tabular}

Shown are the population (in \%), the mean number of H-bonds $\langle\mathrm{HB}\rangle$ the mean hydrophobic core radius gyration $\left\langle R_{g}^{\text {core }}\right\rangle$, as well as the mean dihedral angles $(\phi, \psi)$ (in degrees) of the sixteen peptide groups.

TABLE II. Population Probabilities of the Unfolded State U, the Globule State H, the Partial Folded State P, and the Folded State F Are Listed for Various Temperatures

\begin{tabular}{lcccc}
\hline \multirow{2}{*}{$\begin{array}{l}\text { Temperature } \\
\text { (K) }\end{array}$} & \multicolumn{4}{c}{ Population $(\%)$} \\
\cline { 2 - 5 } & U state & H state & P state & F state \\
\hline 300 & 3.5 & 11.3 & 3.9 & 55.9 \\
310 & 3.3 & 15.5 & 3.9 & 52.3 \\
332 & 3.9 & 20.8 & 6.6 & 44.0 \\
362 & 4.2 & 35.1 & 6.5 & 32.3 \\
389 & 4.5 & 43.4 & 7.6 & 24.7 \\
\hline
\end{tabular}

the $\mathrm{P}$ state has a significantly higher population than the $\mathrm{H}$ state. The large population of the partially folded state $\mathrm{P}$ led them to the speculation that the final hydrophobic core and the $\beta$-strand $\mathrm{H}$-bonds appear nearly simultaneously. As seen from Table II, the population of state $\mathrm{P}$ is lower than state $H$, therefore suggesting that the hydrophobic cores collapse before the H-bonds form. This sequence agrees with the results of Pande and Garcia and their coworkers. $^{5,10}$

To see if the formation of the turn occurs before or after the $\mathrm{H}$-bonds form, we have calculated the average fraction of the native H-bonds as a function of temperature (Fig. 3). H-bonds 1-7 are numbered from the tail to the turn and correspond to the pairs of residues $(1,16),(2,15),(3,14)$, $(4,13),(5,12),(6,11)$, and $(7,10)$. The H-bond 1 is closest to the tail, whereas 7 is closest to the turn. The gradual decrease of the H-bond probability with temperature indicates that there is no sharp transition in the H-bonds. In agreement with Zhou et al., ${ }^{11}$ bonds 2, 3, 4, and 5 are the most stable. The participation of bond 7 is significantly different from Zhou et al., who reported that this bond is almost unstructured, but our results are in reasonable agreement with Garcia and Sanbonmatsu ${ }^{10}$ and Klimov and Thirumalai. ${ }^{21}$ Because the population of bond 7 is lower than others, the turn is expected to form after the H-bonds.

Because the free energy landscape of the $\beta$-hairpin exhibits four clearly distinguishable states, it seems that system shows folding with intermediates. However, the free energy of states $\mathrm{P}, \mathrm{H}$, and $\mathrm{U}$ with respect to the folded state are quite similar: $\Delta G_{\mathrm{P}}=6.69 \mathrm{~kJ} / \mathrm{mol}, \Delta G_{\mathrm{H}}=4.03$ $\mathrm{kJ} / \mathrm{mol}$, and $\Delta G_{\mathrm{U}}=7.0 \mathrm{~kJ} / \mathrm{mol}$. Neglecting the ultrafast (and therefore hardly observable) initial hydrophobic core collapse from $\mathrm{U} \rightarrow \mathrm{H}$, as well as the only weakly populated state $\mathrm{P}$, we may consider $\mathrm{F}$ and $\mathrm{H}$ as the main minima corresponding to the folded and unfolded states. In this sense, the free energy landscape of the $\beta$-hairpin is consistent with a two-state folder with a broad transition state.

The question of the role of helices as folding intermediates of $\beta$-hairpins is still under debate. The authors of refs. $5,6,12,14$, and 16-18 have not found significant helix content, while a high helix content was found at low temperatures for the AMBER94 force field ${ }^{10}$ and the OPLS united-atom force field with implicit solvent. ${ }^{5}$ Here, we reexamine this problem, using the DSSP program ${ }^{47}$ for the calculation of the fraction of $\beta$ and $\alpha$ structures. Figure 4 shows the average number of residues in the $\beta$-sheet and the $\alpha$-helix. At biologically relevant temperatures the $\beta$-sheet content is more than $50 \%$, and it decreases, as expected, with increasing temperature. The $\alpha$-helix content is higher than $3_{10}$ helix, and their sum remains low $(\leq 2 \%)$ at all temperatures (Zhou et al. ${ }^{11}$ reported $1-2 \%$ ). Thus, in accord with the experiments ${ }^{2-4}$ and most all-atom 


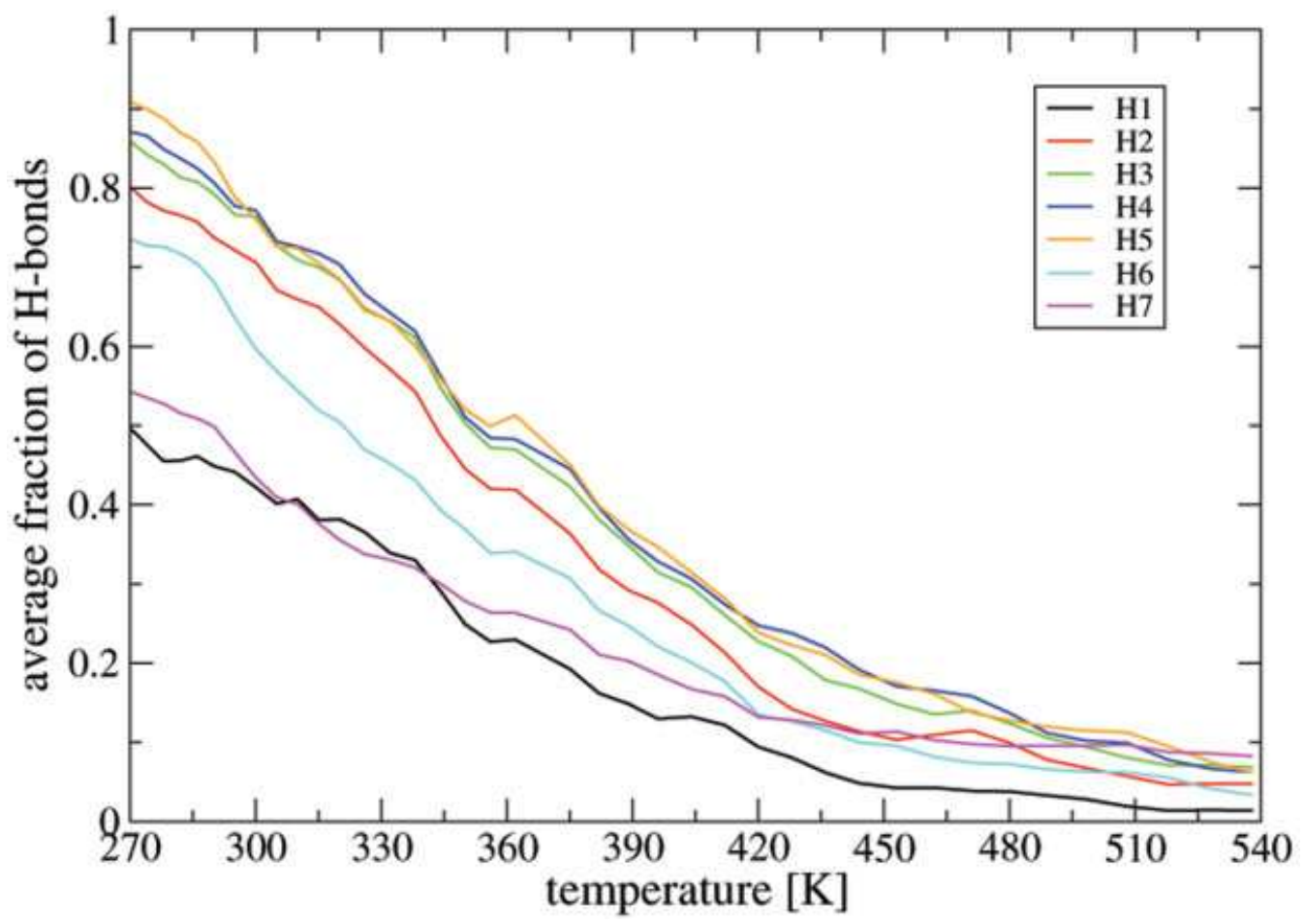

Fig. 3. The average fraction of $\mathrm{H}$ bonds as a function of temperature. The $\mathrm{H}$ bond is said to be formed if the distance between the backbone carbonyl oxygen $\mathrm{CO}$ and the amide hydrogen $\mathrm{NH}$ group is less than $3.5 \AA$.

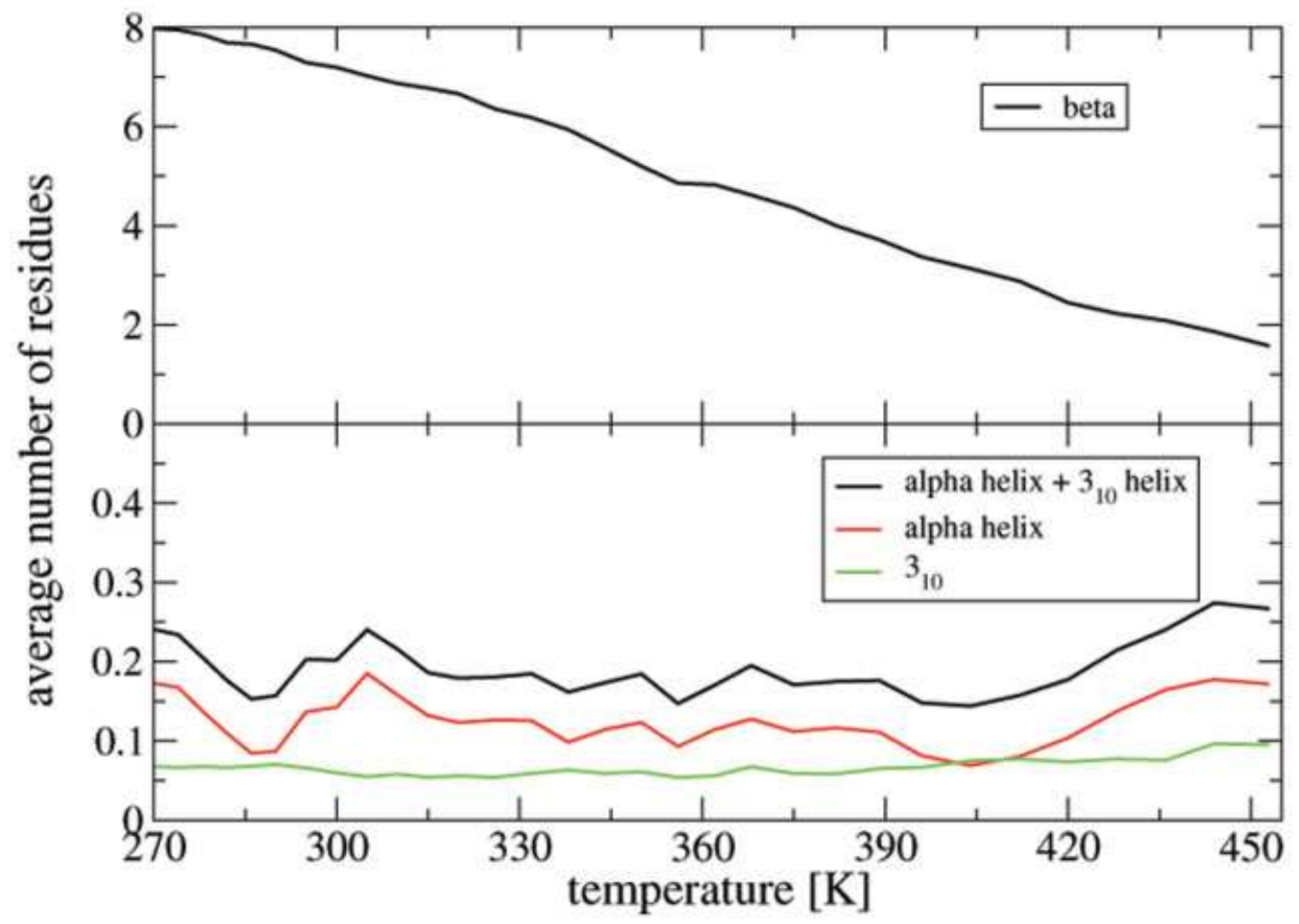

Fig. 4. Average number of residues in $\beta$-sheet (upper panel) and in $\alpha$ and $3_{10}$ helix, as well as their sum (lower panel), shown as a function of temperature. 
simulations, , 5,6,12,16,14,17,18 our result indicates that the $\beta$-hairpin folds without helical intermediates.

In summary, the analysis of the $\left(R_{g}{ }^{\text {core }}, \mathrm{H}\right.$-bond $)$ free energy map suggests (1) that the folding of the $\beta$-hairpin occurs as the sequence: collapse of hydrophobic core $\rightarrow$ formation of $\mathrm{H}$-bond $\rightarrow$ formation of the turn, (2) that the folding exhibits two-state behavior with a broad transition state, and (3) that the helical content of the peptide is very low at any stage of the folding process.

As an alternative representation, Figure 5 shows the dependence of the free energy landscape on the RMSD and the energy $E$ at $T=300 \mathrm{~K}$. The results are reminiscent to the data of Irbäck et al. ${ }^{48}$ obtained for an implicit model with sequence-based potentials. There are three minima corresponding to the three main states referred to as $\mathrm{F}^{\prime}$, $\mathrm{H}^{\prime}$, and $\mathrm{U}^{\prime}$. These states differ mainly in the RMSD, while their energy does not vary significantly. This may be due to the balance between the intramolecular interactions of the peptide and the intermolecular peptide-solvent interactions.

Table I compares the main conformational states of the free-energy surfaces $\Delta G$ (H-bond, $R_{g}{ }^{\text {core }}$ ) and $\Delta G$ (RMSD, $E$ ) shown in Figures 2 and 5. Quite similar to the state F, the state $\mathrm{F}^{\prime}$ has $4.7 \mathrm{H}$-bonds and $R_{g}{ }^{\text {core }} \approx 5.5 \AA$ and similar structures of all residues. The high population of the $\beta$-structure in state $\mathrm{F}^{\prime}(83 \%)$ again shows that the folded state is the most probable one at low temperatures. Both states $\mathrm{H}^{\prime}$ and $\mathrm{U}^{\prime}$ are similar to the state $\mathrm{H}$ in terms of the mean number of H-bonds and $R_{g}{ }^{\text {core }}$. However, a comparison of the $(\phi, \psi)$ angles reveals that state $\mathrm{H}^{\prime}$ corresponds to state $\mathrm{H}$, whereas state $\mathrm{U}^{\prime}$ is quite different. For example, for the residues Phe12, Thr13, and Val14 we find $\alpha$-helical conformations for the state $\mathrm{U}^{\prime}$ and extended conformations for state $\mathrm{H}$ and state $\mathrm{H}^{\prime}$. Furthermore, because the free energies of states $\mathrm{H}^{\prime}$ and $\mathrm{U}^{\prime}$ (relative to $\mathrm{F}$ ) are 2.53 and $6.75 \mathrm{~kJ} / \mathrm{mol}$, respectively, we suggest that states $\mathrm{F}^{\prime}$ and $\mathrm{H}^{\prime}$ correspond to the folded state $\mathrm{F}$ and the unfolded state $\mathrm{H}$ of the $\left(R_{g}{ }^{\text {core }}, \mathrm{H}\right.$-bonds) map, respectively. The states $\mathrm{U}^{\prime}$ and $\mathrm{U}$ are similar due to their high helical content, although they differ in their mean $(\phi, \psi)$ angles. From this analysis, we find that the (RMSD-E) map supports the two-state folding mechanism, but it does not provide the detailed folding pathways as revealed by the $\left(R_{g}{ }^{\text {core }} \mathrm{H}\right.$-bonds) map.

\section{Principal Component Analysis}

Figure 6 shows the free energy map as a function of the first two principal components $v_{1}$ and $v_{2}$ obtained from the (A) Cartesian and (B) dihedral principal component analysis (cPCA and APCA, see Methods), respectively. The properties of the most prominent conformational states of these free energy landscapes are listed in Table III, referred to as S1-S5 for the cPCA states and $\mathrm{S} 1^{\prime}-\mathrm{S} 5{ }^{\prime}$ for the dPCA states. As an illustration, Figure 6 also show representative molecular structures corresponding to these states.

The cPCA yields a relatively well-structured free energy landscape, exhibiting one pronounced minimum and several shallow minima. The free energies of the states $\mathrm{S} 2, \mathrm{~S} 3$, S4, and S5 (relative to S1) are 7.54, 7.37, 8.79, and 9.85
$\mathrm{kJ} / \mathrm{mol}$, respectively. The $\mathrm{dPCA}$, on the other hand, gives a somewhat more complicated picture of the conformational distribution. In addition to the broad global minimum $\mathrm{S}^{\prime}$, we observe four deep minima S2', S3', S4', and S5' with free energies 8.7, 7.69, 6.36, and $8.46 \mathrm{~kJ} / \mathrm{mol}$, respectively. A comparison of the states obtained from the cPCA and the dPCA in terms of $\left\langle R_{g}\right.$ core $\rangle$ and $\langle\mathrm{HB}\rangle$ yields the correspondence (1) $\mathrm{S} 1 \leftrightarrow \mathrm{S}^{\prime}$; (2) $\mathrm{S} 2 \leftrightarrow \mathrm{S} 22^{\prime}$; (3) $\mathrm{S} 3 \leftrightarrow \mathrm{S} 3{ }^{\prime}, \mathrm{S} 4{ }^{\prime}$; and (4) $\mathrm{S} 4 \leftrightarrow \mathrm{S} 5, \mathrm{~S} 5{ }^{\prime}$. A closer inspection, though, reveals differences with respect to some $(\phi, \psi)$ angles: State S3 and S3' are different at residues Asp6, Asp7, and Thr9. State S3 differs from state $\mathrm{S}^{\prime}$ at residues Asp6, Asp7, and Thr11. In the unfolded region, state S4 differs from state S5' mainly at residues Tyr5, Asp7, Thr9, Lys10, and Phe12, while states S5 and S5' are different at residues Glu2, Asp6, Thr13, and Thr15.

We now compare the conformational states obtained from the cPCA and DPCA in Figure 6 to the states revealed by the reaction coordinates $R_{g}$ core and H-bonds in Figure 2 . The following correspondence is observed: (1) $\mathrm{S} 1, \mathrm{~S} 1^{\prime} \leftrightarrow \mathrm{F}$, (2) $\mathrm{S} 2, \mathrm{~S} 2{ }^{\prime} \leftrightarrow \mathrm{P},(3) \mathrm{S} 3, \mathrm{~S} 3{ }^{\prime}, \mathrm{S} 44^{\prime} \leftrightarrow \mathrm{H}$, and (4) S4, S5, S5' $\leftrightarrow$ U. Interestingly, a detailed comparison of the backbone dihedral angles shows that the states obtained from the ( $R_{g}{ }^{\text {core }}, \mathrm{H}$-bonds) map are more similar to the dPCA states than to the cPCA states. For example, the state $\mathrm{U}$ of the $\left(R_{g}{ }^{\text {core }}, \mathrm{H}\right.$-bonds) map differs from the unfolded state $\mathrm{S} 4$ of the cPCA map mainly at residues Tyr5, Asp7, Ala8, Thr9, Lys10, and Phe12, while it only differs from unfolded state S5' of dPCA only at residue Ala8. By using internal coordinates in a suitable way, the APCA is thus able to provide a two-dimensional free energy landscape containing correctly folded $(\mathrm{F})$, partially folded $(\mathrm{P})$, globule $(\mathrm{H})$, and the unfolded $(\mathrm{U})$ states, which largely agree with the states obtained from the $\left(R_{g}{ }^{\text {core }}, \mathrm{H}\right.$-bond $)$ map.

Having compared the states in term of structural quantities, now we compare them in terms of the free energy. Similar to the state $\mathrm{H}$ of the $\left(R_{g}{ }^{\text {core }}, \mathrm{H}\right.$-bonds) map, the state $\mathrm{S} 4{ }^{\prime}$ of the dPCA map has the lowest free energy in the unfolded region. However, because the state $\mathrm{H}$ is lower than the state $\mathrm{S}^{\prime}$, the two-state picture drawn by the two states $\mathrm{F}$ and $\mathrm{H}$ of the $\left(R_{g}{ }^{\text {core }}\right.$,H-bonds) map is more clear than that drawn by the states $\mathrm{S}^{\prime}$ and $\mathrm{S}^{\prime}$ ' of the dPCA map. The cPCA map, on the other hand, is quite smooth in the unfolded part. The state $\mathrm{S} 3$, which is similar to the states $\mathrm{H}_{\text {and }} \mathrm{H}^{\prime}$ in terms of structure, is separated from the states S2 and S4, S5 by very low free energy barriers. Overall, this map does not show a clear two-state folding picture. This can be explained by the fact that the cPCA may suffer from a mixing between internal and overall motion, especially for the unfolded states, which are involved in large-amplitude motions of the molecule. Consequently, the cPCA cannot clearly resolve the unfolded states.

To summarize our analysis of various free energy landscapes, we have found that: (1) The $\left(R_{g}{ }^{\text {core }}, \mathrm{H}\right.$-bonds) map clearly provides the folding pathways as well as the two-state folding picture. (2) The (RMSD-E) map supports the two-state folding mechanism but it does not provide the folding pathway. (3) The cPCA map cannot clearly resolve the unfolded states due to the mixing between 


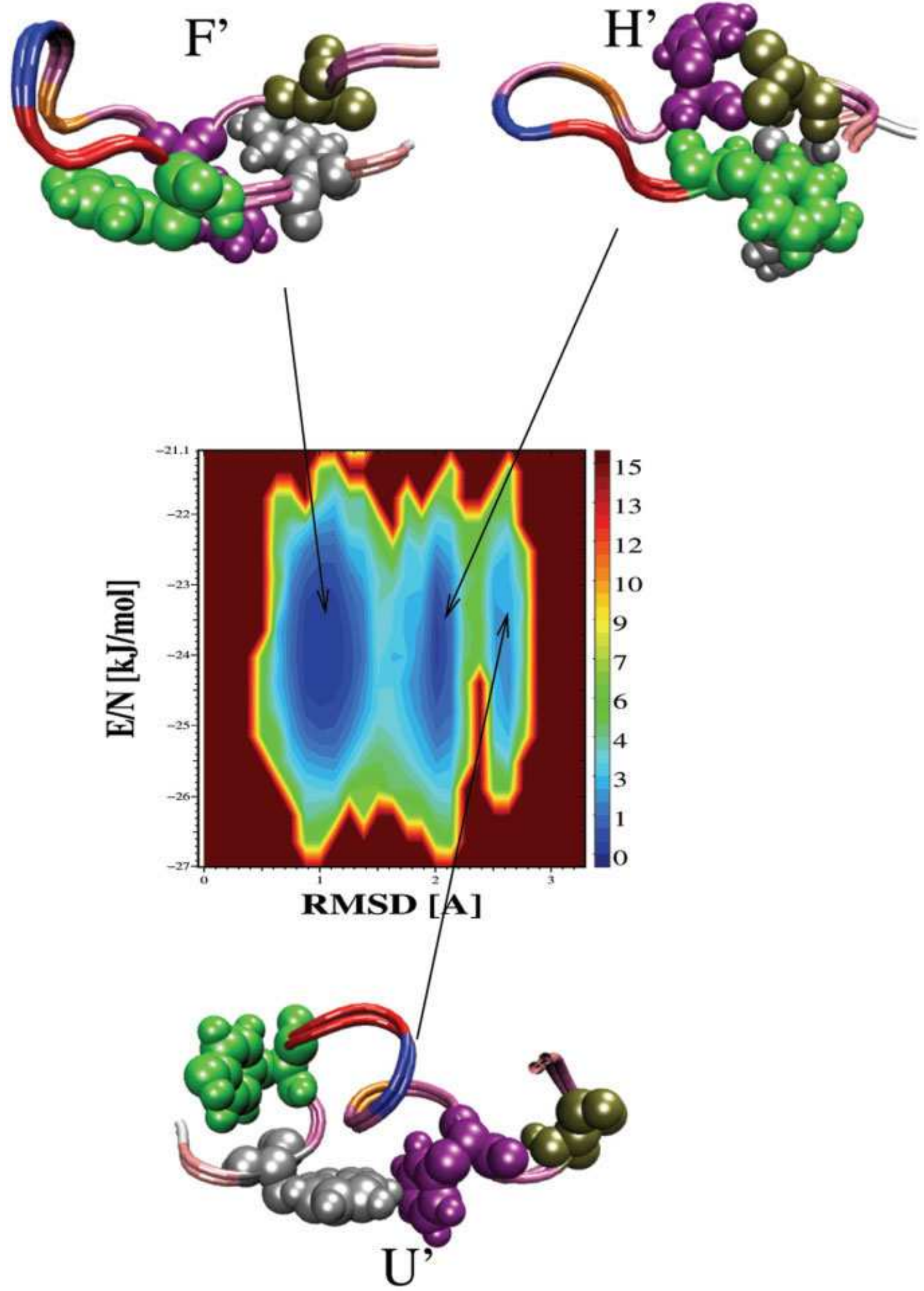

Fig. 5. Free energy map (inkJ/mol) of the $\beta$-hairpin at $300 \mathrm{~K}$, plotted as a function of the enthalpic energy $E$ (divided by the number of atoms) and the RMSD from native state. Representative molecular structures corresponding to the main minima are shown as well. 

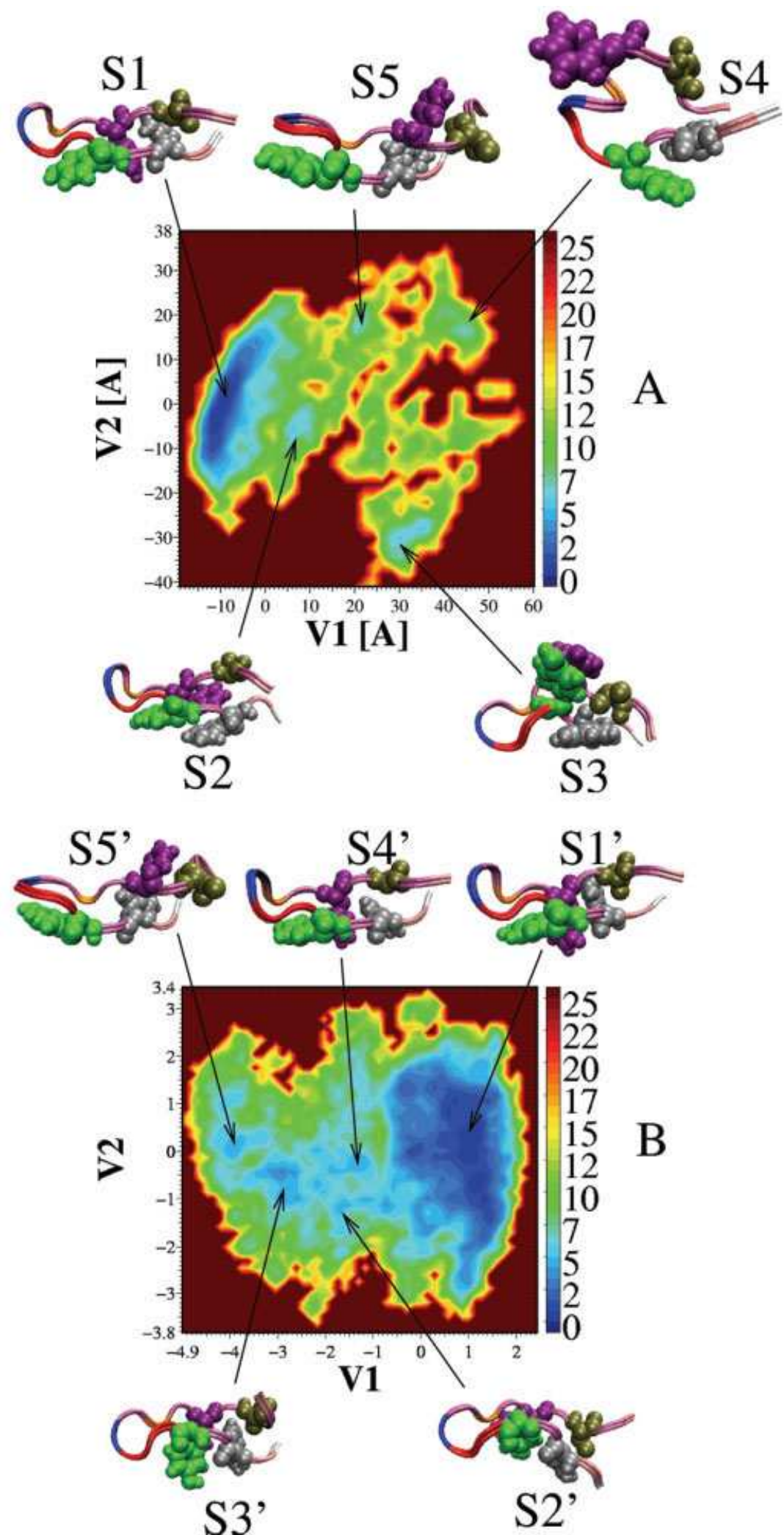

Fig. 6. Free energy map (in $\mathrm{kJ} / \mathrm{mol}$ ) of the $\beta$-hairpin at $300 \mathrm{~K}$, plotted as a function of the first two principal components as obtained from the (A) Cartesian and (B) dihedral principal component analysis, respectively. 
TABLE III. Comparison of the Main Conformational States Obtained from the Cartesian and Dihedral Principal Component Analysis Presented in Figure 6(A) and $(B)$, respectively

\begin{tabular}{|c|c|c|c|c|c|c|c|c|c|c|}
\hline \multirow{5}{*}{$\begin{array}{l}\text { Map } \\
\text { State } \\
\text { Population }(\%) \\
\langle\mathrm{HB}\rangle \\
\left\langle R_{g}^{\text {core }}\right\rangle \\
\end{array}$} & \multicolumn{5}{|c|}{$\mathrm{cPCA}$} & \multicolumn{5}{|c|}{ dPCA } \\
\hline & S1 & $\mathrm{S} 2$ & S3 & $\mathrm{S} 4$ & S5 & $\mathrm{S} 1^{\prime}$ & $\mathrm{S} 2^{\prime}$ & $\mathrm{S} 3^{\prime}$ & $\mathrm{S} 4^{\prime}$ & S5' \\
\hline & 57.2 & 2.8 & 3.0 & 1.7 & 0.9 & 65.1 & 2.0 & 3.0 & 5.1 & 2.2 \\
\hline & 5.1 & 2.7 & 0.2 & 0.2 & 0.0 & 5.1 & 2.1 & 0.9 & 0.3 & 0.1 \\
\hline & 5.6 & 4.9 & 5.1 & 7.3 & 7.5 & 5.5 & 5.4 & 6.0 & 5.5 & 7.0 \\
\hline Gly1 & $24,-28$ & $25,-26$ & $-24,68$ & 5,1 & $-103,102$ & $23,-28$ & 5,12 & 10,19 & $15,-20$ & $-66,55$ \\
\hline Glu2 & $-102,127$ & $-98,124$ & $-80,106$ & $-102,113$ & 4,98 & $-100,123$ & $-100,116$ & $-92,109$ & $-97,120$ & $-52,101$ \\
\hline Trp3 & $-85,107$ & $-89,107$ & $-122,148$ & $-88,113$ & $-94,101$ & $-88,108$ & $-95,115$ & $-93,131$ & $-89,111$ & $-104,121$ \\
\hline Thr4 & $-116,137$ & $-109,132$ & $-66,98$ & $-87,129$ & $-105,137$ & $-111,133$ & $-104,123$ & $-83,116$ & $-110,133$ & $-87,114$ \\
\hline Tyr5 & $-89,130$ & $-95,131$ & $-74,134$ & $-96,-30$ & $-69,103$ & $-94,128$ & $-93,111$ & $-91,66$ & $-94,126$ & $-88,116$ \\
\hline Asp6 & $-103,115$ & $-106,113$ & 3,122 & $-91,150$ & $-100,-61$ & $-103,113$ & $-97,116$ & $-76,122$ & $-101,111$ & $-70,44$ \\
\hline Asp7 & $-62,-39$ & $-61,-40$ & $-78,8$ & $-50,-43$ & $-117,83$ & $-62,-38$ & $-63,-30$ & $-63,-14$ & $-63,-35$ & $-95,58$ \\
\hline Ala8 & $-65,-45$ & $-63,-45$ & $-65,-43$ & $-68,-46$ & $47,-39$ & $-63,-45$ & $-62,-44$ & $-73,-43$ & $-61,-44$ & $-19,-31$ \\
\hline Thr9 & $-85,-3$ & $-85,-2$ & $-91,-44$ & $-71,-24$ & $-93,131$ & $-85,-7$ & $-85,-6$ & $-89,25$ & $-85,-6$ & $-96,54$ \\
\hline Lys10 & 34,47 & 37,44 & $-21,90$ & $-60,-27$ & $-95,94$ & 37,46 & 12,44 & $-66,33$ & 32,46 & $-102,100$ \\
\hline Thr11 & $-118,-28$ & $-119,-26$ & $-92,68$ & $-74,1$ & $-111,102$ & $-120,-28$ & $-113,12$ & $-95,19$ & $-118,-20$ & $-94,55$ \\
\hline Phe12 & $-93,109$ & $-97,112$ & $-91,139$ & $-72,-35$ & $-78,139$ & $-96,110$ & $-91,96$ & $-81,62$ & $-96,109$ & $-84,124$ \\
\hline Thr13 & $-116,128$ & $-111,119$ & $-72,95$ & $-121,127$ & $-79,-24$ & $-113,122$ & $-111,116$ & $-105,83$ & $-112,119$ & $-75,28$ \\
\hline Val14 & $-83,108$ & $-85,110$ & $-116,135$ & $-71,131$ & $-105,136$ & $-86,108$ & $-90,109$ & $-92,107$ & $-88,109$ & $-109,124$ \\
\hline Thr15 & $-97,96$ & $-98,88$ & $-66,57$ & $-60,77$ & $-70,-9$ & $-97,96$ & $-90,82$ & $-64,70$ & $-96,92$ & $-66,11$ \\
\hline Glu16 & $-80,-58$ & $-80,-58$ & $-89,-56$ & $-94,-58$ & $-97,-57$ & $-82,-58$ & $-84,-58$ & $-95,-58$ & $-80,-58$ & $-93,-58$ \\
\hline
\end{tabular}

Shown are the population (in \%), the mean number of $\mathrm{H}$-bonds $\langle\mathrm{HB}\rangle$, the mean hydrophobic core radius gyration $\left\langle R_{g}^{\text {core }}\right\rangle$, as well as the mean dihedral angles $(\phi, \psi)$ (in degrees) of the 16 peptide groups.

internal and overall motion. (4) The dPCA map, in which the problem associated with overall motions is avoided, clearly shows the folding pathway, which is resolved by the $\left(R_{g}{ }^{\text {core }}, \mathrm{H}\right.$-bonds) map. However, the two-state folding picture is a bit less evident, because the dPCA does not explicitly account for the H-bonds, which play an important role in the folding process.

\section{Estimation of Folding Times}

Due to the way the generalized ensemble is constructed, the time dependence of any quantity is lost in the REMD simulation, and therefore the folding time cannot be directly determined from the simulated trajectories. As a remedy, we adopt a diffusion theory due to Wolynes and coworkers, ${ }^{49}$ which allows one to estimate the folding time from the free energy landscape of the system. For a given free energy curve $\Delta G\left(v_{1}\right)$ along the reaction coordinate $v_{1}$, this formulation yields the folding time

$$
\tau_{\text {fold }}=\frac{1}{D\left(v_{1}\right)} \int_{v_{1 u}}^{v_{1 f}} d v_{1} \int_{0}^{v_{1}} d v_{1}^{\prime} \exp \left\{\left[\Delta G\left(v_{1}\right)-\Delta G\left(v_{1}^{\prime}\right)\right] / k_{B} T\right\},
$$

where $D\left(v_{1}\right)$ is the diffusion coefficient in configurational space.

Following Garcia and Sanbonmatsu, ${ }^{10}$ we use the first principal component obtained from the cPCA as reaction coordinate (see Fig. 7). We determine the folding time at $T=300 \mathrm{~K}$, at which most of the experiments have been performed. At this temperature, the minima of the profile at $v_{1} \approx-10$ and $30 \AA$ correspond to the folded and the globule state, respectively. Assuming that the free energy landscape is well approximated by a parabolic potential around the globule state, the diffusion coefficient can be approximated by $D\left(v_{1}\right)=\left(\Delta v_{1}\right)^{2} / \tau_{\text {corr }}$, where $\left(\Delta v_{1}\right)^{2}$ and $\tau_{\text {corr }}$ are the variance and the autocorrelation time of the

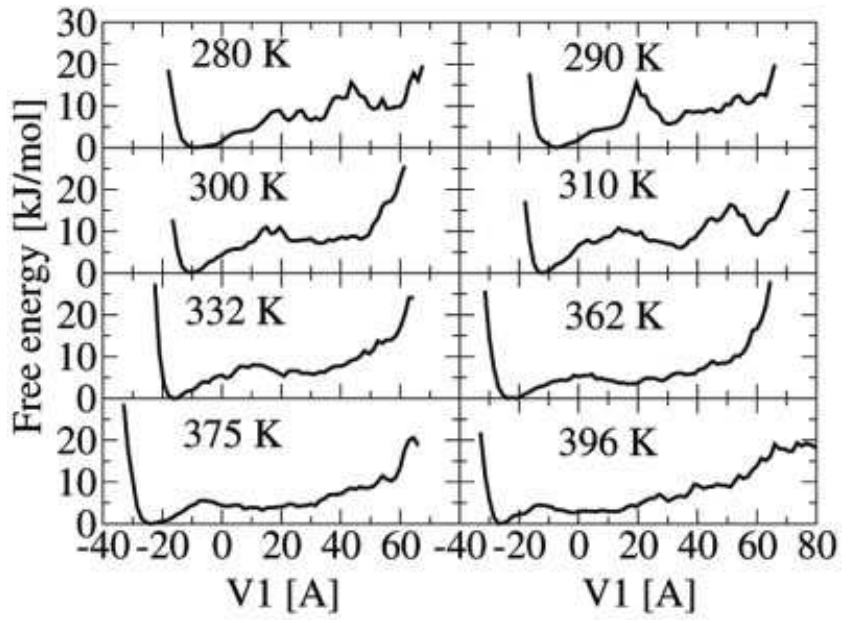

Fig. 7. Free energy landscape of the $\beta$-hairpin drawn as a function of the first principal component at various temperatures.

reaction coordinate $v_{1}$, respectively. These quantities are estimated from a standard (not REMD) simulation at constant (N,V,T) for $15 \mathrm{~ns}$ at $T=300 \mathrm{~K}$. We obtained $\tau_{\text {corr }} \approx 1.1 \mathrm{~ns},\left(\Delta v_{1}\right)^{2} \approx 111 \AA^{2}$ and $D\left(v_{1}\right)=101 \AA^{2} / \mathrm{ns}$. By evaluating Equation (9), we thus obtain $\tau_{\text {fold }} \approx 6.9 \mu \mathrm{s}$. Because the validity of the underlying assumptions (use of a single reaction coordinate and the quasi-harmonic approximation) are difficult to assess, this folding time can only be considered as a rough estimate. Nevertheless, the result is in good agreement with the experimental ${ }^{4,20}$ and other simulation results. ${ }^{9,10}$ For example, Eaton and coworkers ${ }^{4}$ reported $\tau_{\text {fold }}=6 \mu \mathrm{s}$ from temperature-jump fluorescence experiments, Zagrovic et al. obtained $\approx 4.7 \mu \mathrm{s}$ by using a GB/SA implicit solvent model, and Garcia and Sanbonmatsu ${ }^{10}$ reported $\tau_{\text {fold }}=1-7 \mu$ s obtained from AMBER94 simulations with explicit solvent. 
(a)

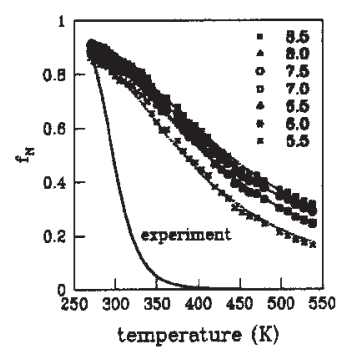

(b)

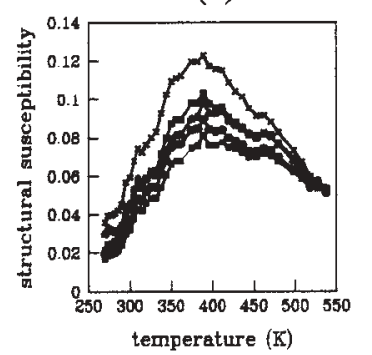

(c)

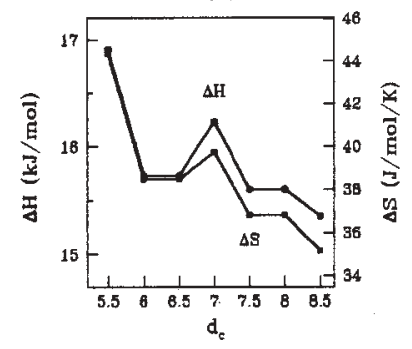

Fig. 8. (a) Temperature dependence of the fraction of native contacts $f_{N}$ obtained for various values of the cutoff distance $d_{c}$. The native hairpin population (solid curve) was calculated by using experimental thermodynamic parameters $\Delta H=48.5 \mathrm{~kJ} \mathrm{~mol}^{-1}$ and $\Delta S=163.2 \mathrm{~J} / \mathrm{mol} / \mathrm{K} .{ }^{4}(\mathbf{b})$ The temperature dependence of the structural susceptibility $\Delta$. For all values of $d_{C}, \Delta$ exhibits a peak at $T_{F}=389 \mathrm{~K}$. (c) Dependence of $\Delta H$ and $\Delta S$ on the cutoff distance $d_{c}$, obtained by fitting the temperature dependences of the fraction of native contracts shows in (a) in a two-state model.

The existence or absence of intermediates that may slow down the folding process depends on the force fields. The fact that all of the force fields provide the compatible estimate for the folding time at the room temperature suggests that the helix intermediates play a minor role. It can be seen, for example, from Figure 5(a) of ref. 10, at $T=$ 270 and $282 \mathrm{~K}$ they correspond to four basins in the free energy landscape plotted as a function of $v_{1}$, but the corresponding barriers are shallow.

\section{Folding Thermodynamics}

Let us finally study the temperature dependence of the folding transition as monitored by various reaction coordinates. We first try to estimate the folding temperature from the free energy landscape as a function of the first principal component of the cPCA (Fig. 7). At $280 \mathrm{~K}$ the free energy profile exhibits two well-defined minima. With increasing temperature, the width of the minima and of the free energy profile in general is found to increase. Also, the barriers become lower and essentially vanish at $362 \mathrm{~K}$. This is in agreement with Zhou et al., ${ }^{11}$ who have also observed the crossover behavior of the free energy landscape at $360 \mathrm{~K}$ and identified it as the folding temperature $T_{F}$. It is shown below, however, that the folding temperature defined from the maximum of the structural susceptibility is higher than the folding temperature estimated from the free energy profiles.

We next consider the temperature dependence of the fraction of native contacts $\langle\chi\rangle$, calculated from Equation (7). $\langle\chi\rangle$ is equivalent to the probability $f_{\mathrm{N}}$ of being in the native basin, which may be measured in experiments. ${ }^{4}$ Figure 8(a) shows this quantity obtained for various values of the cutoff distance $d_{c}$. The effect of cutoff distance $d_{c}$ is clearly visible at high temperatures. In agreement with experiment, ${ }^{4} f_{\mathrm{N}}$ is about 0.9 at $T=270 \mathrm{~K}$. Increasing the temperature, however, the departure from the experimental curve becomes significant. Nevertheless, our data seem to fit experiments better than those obtained from the OPLS-AA force field ${ }^{11}$ and various implicit solvent models (OPLS-AA/SGB, AMBER94/GBSA, AMBER96/ GBSA, and AMBER99/GBSA). ${ }^{50}$ The best agreement of $\langle\chi\rangle$ with experimental data was obtained by Klimov and Thirumalai ${ }^{21}$ for a minimalist off-lattice model.
Figure 8(b) shows the temperature dependence of the structural susceptibility $\Delta$ defined in Equation (8). Interestingly, the position of peak does not depend on the cutoff distance $d_{c}$. It corresponds to the folding temperature $T_{F}=$ $389 \mathrm{~K}$, which is clearly higher than the experimental value $T_{F}=297$ reported by Munoz et al. ${ }^{4}$ Depending on the employed model, a large variety of folding temperature has been reported for the $\beta$-hairpin, ranging from $335 \mathrm{~K}$ for the CHARMM force field ${ }^{6}$ to $485 \mathrm{~K}$ for the OPLS-AA/AGBNP force field. ${ }^{15}$ Defining the melting temperature as the point at which the free energy landscape becomes smooth, Zhou et al. ${ }^{11}$ obtained $T_{F}=362 \mathrm{~K}$ using the OPLS-AA force field, whereas Garcia and Sanbonmatsu ${ }^{10}$ found $T_{F}=330 \mathrm{~K}$ using AMBER94, which is similar to our result in Figure 7.

To estimate the changes in enthalpic energy $\Delta H$ and entropy $\Delta S$ at the folding transition, we fit the temperature dependence of $f_{\mathrm{N}}=\langle\chi\rangle$ shown in Figure 8(a) to a two-state model, that is,

$$
f_{N}=\frac{1}{1+\exp \left(-(\Delta H-T \Delta S) / k_{B} T\right)} .
$$

The fact that the two-state fit works well for all values of $d_{c}$ [see Fig. 8(a)] indicates that the $\beta$-hairpin can be considered as a two-state folder. We note that this analysis take into account all degrees of freedom of the system. Although the temperature dependence of the free energy landscape (e.g., in Figs. 2 and 7) leads to the same conclusion, it should be stressed that the somewhat ambiguous choice and the limited number of considered reaction coordinates renders the latter analysis less stringent.

Depending on the cutoff distance $d_{c}, \Delta H$ varies between 15.5 and $16.7 \mathrm{~kJ} / \mathrm{mol}$ and $\Delta S$ between 35.1 and $44.6 \mathrm{~J} /(\mathrm{mol}$ K) [see Fig. 8(c)]. The averaged values are $\Delta H=15.9 \pm 0.5$ $\mathrm{kJ} / \mathrm{mol}$ and $\Delta S=38.5 \pm 2.8 \mathrm{~K}$ ). They are clearly lower than the experimental estimate $\Delta H=48.5 \mathrm{~kJ} / \mathrm{mol}$ and $\Delta S=$ $163.2 \mathrm{~J} / \mathrm{mol} / \mathrm{K}$ reported in ref. 4. Compared to other simulation studies, we find that our result for $\Delta H$ is close to the value of $18.1 \mathrm{~kJ} / \mathrm{mol}$ quoted by Dinner et al. ${ }^{6}$ but lower than $29.3 \mathrm{~kJ} / \mathrm{mol}$ obtained by Klimov and Thirumalai. ${ }^{21}$ Furthermore, our results for the entropic contribution to the free energy are compatible with $T_{F} \Delta S=16.4$ $\mathrm{kJ} / \mathrm{mol}$ of Dinner et al. 
Let us finally discuss possible reasons for the deviations between experiment and theory when studying the thermodynamics properties of the peptide. As explained above, this finding is the rule rather than the exception, for example, classical MD simulations typically find a significantly higher melting temperature than obtained in experiment. First, because most studies employ the folded structure as in the initial state of the simulation, this may be a consequence of still incomplete sampling. Starting from random initial structures as done in refs. 17 and 18 could be a remedy to avoid the possible overestimation of the native state. Second, as discussed by Zhou et al., ${ }^{11}$ a central issue is certainly the force field parameterization of the peptide as well as of the explicit water solvent. This is, because the fitting of force field parameters is performed for room temperature rather than for the high temperature range. For example, van Gunsteren and coworkers have shown that the properties of the SPC water model (e.g., diffusion constant and heat capacity) differ significantly from experiment at high temperatures. ${ }^{51}$ Finally, it should be kept in mind, that simple potential-energy models commonly employed in biomolecular force fields represent a severe approximation of the true ab initio energy. For example, cooperative effects due to many-body interactions or the polarizability of atoms are completely neglected in the potential-energy function, and are only accounted for in a mean field-type parameterization of two-body forces with constant charges.

To study the latter issue, we consider a dimensionless measure of cooperativity of the folding-unfolding transi$\operatorname{tion}^{52,53}$

$$
\Omega_{c}=\frac{T_{F}^{2}}{\Delta T}\left|\frac{d f_{N}}{d T}\right|_{T}=T_{F},
$$

where $\Delta T$ is the full width at half-maximum of the function $d f_{\mathrm{N}} / d T$ and $T_{F}$ is the folding transition temperature identified with the maximum in $d f_{\mathrm{N}} / d T$. Using the averaged values of $\Delta H$ and $\Delta S$ and Equation (10) for $f_{\mathrm{N}}$, we obtain $\Omega_{c}$ $\approx 1$.7. Although this cooperativity is higher than the value of 0.82 quoted in ref. 21 , it is still one order of magnitude lower than the experimental result $\Omega_{c} \approx 30.54$ (Interestingly, the experiments in refs. 4 and 23 give almost the same value of $\Omega_{c}$.) Thus, we find that the degree of cooperativity in the hairpin simulations is much lower than the experimental result. As explained above, this may be caused by the neglect of the polarizability and multiparticle interactions, which are presumably required to correctly describe the cooperativity seen in proteins. ${ }^{55,56}$

\section{CONCLUSIONS}

We have presented extensive REMD simulations (40 replicas and $340 \mathrm{~ns}$ total simulation time) of the folding of a 16-residue $\beta$-hairpin peptide using the GROMOS96 force field and the SPC water model. To explore the mechanism as well as the thermodynamics of the folding process, we have studied the free energy landscape as a function of several types of reaction coordinates, including (1) the number of native $\mathrm{H}$-bonds in the $\beta$-hairpin conformation, (2) the radius of gyration of the hydrophobic core $R_{g}{ }^{\text {core }}$, (3) the enthalpic energy $E$, (4) the RMSD from the PDB structure, (5) and the first few principal components.

The $\left(R_{g}{ }^{\text {core }}, \mathrm{H}\right.$-bond $)$ free energy map at $T=300 \mathrm{~K}$ exhibits four local minima corresponding to the folded $(\mathrm{F})$, partially folded (P), molten globule (H), and unfolded (U) states. The L-shape of this free energy representation suggests that the folding of the $\beta$-hairpin occurs as the sequence: collapse of hydrophobic core $\rightarrow$ formation of $\mathrm{H}$-bond $\rightarrow$ formation of the turn. In agreement with experiment and most all-atom simulations, ${ }^{5,6,12,14,16-18}$ the GROMOS96 simulations suggest that the $\beta$-hairpin folds without helical intermediates. Neglecting the ultrafast (and therefore hardly observable) initial hydrophobic core collapse from $\mathrm{U} \rightarrow \mathrm{H}$, as well as the only weakly populated state $\mathrm{P}$, we may consider $\mathrm{F}$ and $\mathrm{H}$ as the main minima corresponding to the folded and unfolded states. In this sense, the free energy landscape of the $\beta$-hairpin is consistent with a two-state behavior with a broad transition state.

Although the ( $R_{g}{ }^{\text {core }}, \mathrm{H}$-bonds) map clearly provides the folding pathways as well as the two-state folding picture, the (RMSD-E) map as well as the free energy map provided by the first two principal components of the cPCA provide only limited information of the folding pathway. In particular, the cPCA map cannot clearly resolve the unfolded states due to the mixing between internal and overall motion. The dPCA map, in which the problem associated with overall motions is avoided, clearly shows the folding pathway which is resolved by the $\left(R_{g}{ }^{\text {core }}\right.$, H-bonds) map. However, the two-state folding picture is a bit less evident, because the dPCA does not explicitly account for the H-bonds, which play an important role in the folding process.

From the temperature dependence of the structural susceptibility we have obtained a folding transition temperature of $389 \mathrm{~K}$, which is higher than the experimental estimate of $297 \mathrm{~K}$. Furthermore, the enthalpy and entropy jumps at the melting temperature are found to be about three times lower than the experimental estimates, indicating that the folding-unfolding transition in silico is less cooperative than its in vitro counterpart. As discussed in some detail, this appears to be a consequence of (1) the neglect of polarizability and many-body interactions, and (2) the fact that force field parameters are derived for room temperature rather than for the high temperature range. Hereby, the temperature dependence of the parameterization of two-body forces arises to effectively account for the interactions not considered in the potential-energy model.

To conclude, the present GROMOS96 REMD simulation appears to give a comprehensive and appropriate description of the $\beta$-hairpin at room temperature. Similar to virtually all force field-based models to date, however, one only obtains an approximate description of the hightemperature range. Nevertheless, one may expect that the model is able to qualitatively characterize the folding and unfolding mechanism of peptides.

\section{ACKNOWLEDGMENTS}

M.S.L. thanks A. Irbäck for useful discussions. 


\section{REFERENCES}

1. Daggett, V, Fersht, AR. Is there a unifying mechanism for protein folding? Trends Biochem Sci 2003;28:18.

2. Blanco, FJ, Rivas G, Serrano L. A short linear peptide that folds in a native stable $\beta$-hairpin in aqueous solution. Nat Struct Biol $1994 ; 1: 584$.

3. Blanco FJ, Serrano L. Folding of protein G B1 domain studied by the conformal characterization of fragments comprising its secondary structure. Eur J Biochem. 1995;230:634.

4. Munoz V, Thompson PA, Hofrichter J, Eaton WA. Folding dynamics and mechanism of $\beta$-hairpin formation. Nature 1997;390:196.

5. Pande VS, Roshkar DS. Molecular dynamics simulations of unfolding and refolding of a $\beta$-hairpin fragment of protein G. Proc Natl Acad Sci USA 1999;96:9062.

6. Dinner AR, Lazaridis T, Karplus M. Understanding $\beta$-hairpin formation. Proc Natl Acad Sci USA 1999;96:1068.

7. Roccatano D, Amadei A, Nola N Di, Berendsen HJ. A molecular dynamics study of the 41-56 $\beta$-hairpin from b1 domain of protein G. Protein Sci 1999;10:2130.

8. Ma B, Nussinov R. Molecular dynamics simulations of a $\beta$-hairpin fragment of protein G: Balance between side-chain and backbone forces. J Mol Biol 2000;296:1091.

9. Zagrovic B, Sorin EJ, Pande VS. $\beta$-Hairpin folding simulation in atomistic detail. J Mol Biol 2001;313:151.

10. Garcia AE, Sanbonmatsu KY. Exploring the energy landscape of a $\beta$-hairpin in explicit solvent. Proteins 2001;42:345

11. Zhou R, Berne BJ, Germain R. The free energy landscape for $\beta$-hairpin folding in explicit water. Proc Natl Acad Sci USA 2001;98:14931.

12. Zhou R, Berne BJ. Can a continuum solvent model reproduce the free energy landscape of a $\beta$-hairpin in water? Proc Natl Acad Sci USA 2002;99:12777.

13. Lee J, Shin S, Two-dimensional correlation analysis of peptide unfolding: molecular dynamics simulations of $\beta$-hairpin. J Phys Chem B 2002;106:8796.

14. Bolhuis PG. Transition-path sampling of $\beta$-hairpin folding. Proc Natl Acad Sci USA 2003;100:12129.

15. Felts AK, Narano Y, Gallicchio E, Levy RM. Free energy surface of $\beta$-hairpin and $\alpha$-helical peptides generated by replica exchange molecular dynamics with the AGBNP implicit solvent model. Proteins 2004;56:310.

16. Zhou R. Exploring the protein folding free energy landscape: coupling replica exchange method with P3ME/RESPA algorithm. J Mol Graph Model 2004;22:451.

17. Yoda T, Sugita Y, Okamoto Y. Comparisons of force fields for proteins by generalized-ensemble simulations. Chem Phys Lett 2004;386:460

18. Yoda T, Sugita Y, Okamoto Y. Secondary-structure preferences of force fields for proteins evaluated by generalized-ensemble simulations. Chem Phys 2004;307:269.

19. Munoz V, Henry ER, Hofrichter J, Eaton WA. A statistical mechanical model for $\beta$-hairpin kinetics. Proc Natl Acad Sci USA 1998;95:5872

20. Du D, Zhu Y, Huang CY, Gai F. Understanding the key factors that control the rate of $\beta$-hairpin folding. Proc Natl Acad Sci USA 2004;101:15915.

21. Klimov DK, Thirumalai D. Mechanisms and kinetics of $\beta$-hairpin formation. Proc Natl Acad Sci USA 2000;97:2544.

22. Honda S, Kobayashi N, Munekata F. Thermodynamics of a $\beta$-hairpin structure: evidence for cooperative formation of folding nucleus. J Mol Biol 2000;295:269.

23. Kobayashi N, Honda S, Yoshii H, Munekata F. Role of side-chains in the cooperative $\beta$-hairpin folding of the short terminal fragment derived from Streptococcal protein G. Biochemistry 2000;39:6564.

24. Swendsen R H, Wang JS. Replica Monte Carlo simulations of spin glasses. Phys Rev Lett 1986;57:2607.

25. Geyer CJ. Practical Markov chain Monte Carlo. Stat Sci 1992;7:473.

26. Hukushima K, Nemoto K. Exchange Monte Carlo method and application to spin glass simulations. J Phys Soc Jpn 1996;65:1604.

27. Tesi MC, van Rensburg EJJ, Orlandini E, Whittington SG. Monte Carlo study of the interacting self-avoiding walk model in three dimensions. J Stat Phys 1996;82:155.

28. Hansmann UHE. Parallel tempering algorithm for conformational studies of biological molecules. Chem Phys Lett 1997;281:140.

29. Sugita Y, Okamoto Y. Replica-exchange molecular dynamics method for protein folding. Chem Phys Lett 1999;314:141.
30. Sugita Y, Okamoto Y. Replica-exchange multicanonical algorithm and multicanonical replica-exchange method for simulating systems with rough energy landscape. Chem Phys Lett 2000;329:261.

31. Mitsutake A, Sugita Y, Okamoto Y. Generalized-ensemble algorithms for molecular simulations of biopolymers. Biopolymers 2001;60:96.

32. van Gunsteren WF, Billeter SR, Eising AA, Hünenberger PH, Krüger P, Mark AE, Scott WRP, Tironi IG. Biomolecular simulation: the GROMOS96 manual and user guide. Zürich: Vdf Hochschulverlag AG an der ETH; 1996.

33. Berendsen HJC, Postma JPM, van Gunsteren WF, Hermans J. Intermolecular Forces. Dortrecht: Reidel; 1981. p 331.

34. Berendsen HJC, van der Spoel D, van Drunen R. GROMACS: a message-passing parallel molecular dynamics implementation. Comp Phys Commun 1995;91:43.

35. Lindahl E, Hess B, van der Spoel D. GROMACS 3.0: a package for molecular simulation and trajectory analysis. J Mol Model 2001;7:306.

36. Ryckaert JP, Cicotti G, Berendsen HJC. Numerical Integration of the Cartesian equations of motion of a system with constraints: molecular dynamics of $n$-alkanes. J Comput Phys 1977;23:327.

37. Darden T, York D, Pedersen L. Particle mesh Ewald: an N $\cdot \log (N)$ method for Ewald sums in large systems. J Chem Phys 1993;98: 10089.

38. Berendsen HJC, Postma JPM, van Gunsteren WF, Dinola A, Haak JR. Molecular dynamics with coupling to an external bath. J Chem Phys 1984;81:3684.

39. Sanbonmatsu KY, Garcia AE. Structure of Met-enkephalin in explicit aqueous solution using replica exchange molecular dynamics. Proteins 2002;46:225.

40. Nguyen PH, Mu Y, Stock G. Structure and energy landscape of a photoswitchable peptide: a replica exchange molecular dynamics study. Proteins 2005;60:485-494.

41. Ichiye T, Karplus M. Collective motions in proteins: a covariance analysis of atomic fluctuations in molecular dynamics and normal mode simulations. Proteins 1991;11:205.

42. Kitao A, Hirata F, Go N. The effects of solvent on the conformation and the collective motions of protein: normal mode analysis and molecular dynamics simulations of melittin in water and in vacuum. Chem Phys 1991;158:447.

43. Garcia AE. Large-amplitude nonlinear motions in proteins. Phys Rev Lett 1992;68:2696.

44. Amadei, Linssen ABM, Berendsen HJC. Essential dynamics of proteins. Proteins 1993; 17:412.

45. Mu Y, Nguyen PH, Stock G. Energy landscape of a small peptide revealed by dihedral angle principle component analysis. Proteins 2005;58:45.

46. Camacho CJ, Thirumalai D. Kinetics and thermodynamics of folding in model proteins. Proc Natl Acad Sci USA 1993;90:6369.

47. Kabsch W, Sander C. Dictionary of protein secondary structure: pattern recognition of hydrogen bonded and geometrical features. Biopolymers 1983;22:2577.

48. Irbäck A, Samuelsson B, Sjunnesson F, Wallin S. Thermodynamics of $\alpha$ - and $\beta$ - structure formation in proteins. Biophys $J$ $2003 ; 85: 1466$.

49. Socci ND, Onuchic JN, P.G. Wolynes. Diffusive dynamics of the reaction coordinate for protein folding funnels. J Chem Phys 1996; 104:5860.

50. Zhou R. Free energy landscape of protein folding in water: explicit vs. implicit solvent. Proteins 2003;53:148.

51. Walser R, Mark AE, van Gunsteren WF. On the temperature and pressure dependence of a range of properties of a type of water model commonly used in high-temperature protein unfolding simulations. Biophys J 2000;78:2752.

52. Klimov DK, Thirumalai D. Cooperativity in protein folding: from lattice models with side chains to real proteins. Fold Des 1998; 3:127.

53. Li MS, Klimov DK, Thirumalai D. Thermal denaturation and folding rates of single domain proteins: sizes matter. Polymer 2004; $45: 573$.

54. Li MS, Klimov DK, Thirumalai D. Finite size effects on thermal denaturation of globular proteins. Phys Rev Lett 2004;93:268107.

55. Kaya H, Chan HS. Solvation effects and driving forces for protein thermodynamics and kinetic cooperativity: how adequate is nativecentric topological modeling? J Mol Biol 2003;326:911.

56. Tsai J, Gerstein M, Levitt M. Simulating the minimum core for hydrophobic collapse in globular proteins. Protein Sci 1997;6: 2606 . 